\title{
RENORMALIZED ENTROPY SOLUTIONS FOR QUASILINEAR ANISOTROPIC DEGENERATE PARABOLIC EQUATIONS
}

\author{
MOSTAFA BENDAHMANE AND KENNETH H. KARLSEN
}

\begin{abstract}
We prove the well posedness (existence and uniqueness) of renormalized entropy solutions to the Cauchy problem for quasilinear anisotropic degenerate parabolic equations with $L^{1}$ data. This paper complements the work by Chen and Perthame [19], who developed a pure $L^{1}$ theory based on the notion of kinetic solutions.
\end{abstract}

\section{INTRODUCTION}

We consider the Cauchy problem for quasilinear anisotropic degenerate parabolic equations with $L^{1}$ data. This convection-diffusion type problem is of the form

$$
\partial_{t} u+\operatorname{div} f(u)=\nabla \cdot(a(u) \nabla u)+F, \quad u(0, x)=u_{0}(x),
$$

where $(t, x) \in(0, T) \times \mathbf{R}^{d} ; T>0$ is fixed; div and $\nabla$ are with respect to $x \in \mathbf{R}^{d}$; and $u=u(t, x)$ is the scalar unknown function that is sought. The (initial and source) data $u_{0}(x)$ and $F(t, x)$ satisfy

$$
u_{0} \in L^{1}\left(\mathbf{R}^{d}\right), \quad F \in L^{1}\left((0, T) \times \mathbf{R}^{d}\right) .
$$

The diffusion function $a(u)=\left(a_{i j}(u)\right)$ is a symmetric $d \times d$ matrix of the form

$$
a(u)=\sigma(u) \sigma(u)^{\top} \geq 0, \quad \sigma \in(C(\mathbf{R}))^{d \times K}, \quad 1 \leq K \leq d,
$$

and hence has entries

$$
a_{i j}(u)=\sum_{k=1}^{K} \sigma_{i k}(u) \sigma_{j k}(u), \quad i, j=1, \ldots, d .
$$

Date: June 2, 2003.

1991 Mathematics Subject Classification. 35K65, 35L65.

Key words and phrases. degenerate parabolic equation, quasilinear, anisotropic diffusion, entropy solution, renormalized solution, uniqueness, existence.

This work was supported by the BeMatA program of the Research Council of Norway and the European network HYKE, funded by the EC as contract HPRN-CT-2002-00282. This work was done while MB visited the Centre of Mathematics for Applications (CMA) at the University of Oslo, Norway, and he is grateful for the hospitality. 
The nonnegativity requirement in (1.3) means that for all $u \in \mathbf{R}$

$$
\sum_{i, j=1}^{d} a_{i j}(u) \lambda_{i} \lambda_{j} \geq 0, \quad \forall \lambda=\left(\lambda_{1}, \ldots, \lambda_{d}\right) \in \mathbf{R}^{d} .
$$

Finally, the convection flux $f(u)$ is a vector-valued function that satisfies

$$
f(u)=\left(f_{1}(u), \ldots, f_{d}(u)\right) \in\left(\operatorname{Liploc}_{\text {loc }}(\mathbf{R})\right)^{d} .
$$

It is well known that (1.1) possesses discontinuous solutions and that weak solutions are not uniquely determined by their initial data (the scalar conservation law is a special case of (1.1)). Hence (1.1) must be interpreted in the sense of entropy solutions $[32,41,42]$. In recent years the isotropic diffusion case, for example the equation

$$
\partial_{t} u+\operatorname{div} f(u)=\Delta A(u), \quad A(u)=\int_{0}^{u} a(\xi) d \xi, \quad 0 \leq a \in L_{\mathrm{loc}}^{\infty}(\mathbf{R}),
$$

has received much attention, at least when the data are regular enough (say $L^{1} \cap L^{\infty}$ ) to ensure $\nabla A(u) \in L^{2}$. Various existence results for entropy solutions of (1.5) (and (1.1)) can be derived from the work by Vol'pert and Hudjaev [42]. Some general uniqueness results for entropy solutions have been proved in the one-dimensional context by Wu and Yin [43] and Bénilan and Touré [6]. In the multi-dimensional context a general uniqueness result is more recent and was proved by Carrillo $[15,14]$ using Kružkov's doubling of variables device. Various extensions of his result can be found in [13, $27,31,29,34,35,39]$, see also [17] for a different approach and [40] for a uniqueness proof for piecewise smooth weak solutions. Explicit "continuous dependence on the nonlinearities" estimates were proved in [20]. There are also several recent studies concerned with the convergence of numerical schemes for $(1.5)$, see $[25,26,30,27,36,35,2,12]$. In the literature just cited it is essential that the solutions $u$ possess the regularity $\nabla A(u) \in L^{2}$. This excludes the possibility of imposing general $L^{1}$ data, since it is well known that in this case one cannot expect that much integrability (see, e.g., the citations below on renormalized solutions).

The general anisotropic diffusion case (1.1) is more delicate and was successfully solved only recently by Chen and Perthame [19]. Chen and Perthame introduced the notion of kinetic solutions and provided a wellposedness theory for (1.1) with $L^{1}$ data. Within their kinetic framework, explicit continuous dependence and error estimates for $L^{1} \cap L^{\infty}$ entropy solutions were obtained in [18]. With the only assumption that the data belong to $L^{1}$, we cannot expect a solution of (1.1) to be more than $L^{1}$. Hence it is in general impossible to make distributional sense to (1.1) (or its entropy formulation). In addition, as already mentioned above, we cannot expect the gradient of the diffusion function to be square-integrable, which seems to be an essential condition for uniqueness. Both these problems were elegantly dealt with in [19] using the kinetic approach. 
The purpose of the present paper is to offer an alternative "pure" $L^{1}$ well posedness theory for (1.1) based on a notion of renormalized entropy solutions and the classical Kružkov method [32]. The notion of renormalized solutions was introduced by DiPerna and Lions in the context of Boltzmann equations [23, 22] (see also [24]). This notion (and a similar one) was then adapted to nonlinear elliptic and parabolic equations with $L^{1}$ (or measure) data by various authors, see for example $[10,11,38,4,3,33,7,9,21,16$, 8] (the list is far from being complete). Benilan, Carrillo, and Wittbold [5] introduced recently a notion of renormalized Kružkov entropy solutions for scalar conservation laws with $L^{1}$ data and proved the existence and uniqueness of such solutions. Their theory generalizes the Kružkov well posedness theory for $L^{\infty}$ entropy solutions [32]. In passing, we mention that an alternative $L^{1}$ theory for scalar conservation laws has been developed by Perthame [37]. He has build up a theory around the notion of kinetic solutions, which is the notion that is generalized to (1.1) in [19].

Motivated by the above literature on renormalized solutions and [19], we introduce herein a notion of renormalized entropy solutions for (1.1) and prove its well posedness. Let us illustrate our notion of an $L^{1}$ solution on the isotropic diffusion equation (1.5) with initial data $\left.u\right|_{t=0}=u_{0} \in L^{1}$. To this end, let $T_{l}: \mathbf{R} \rightarrow \mathbf{R}$ denote the truncation function at height $l>0$ and let $\zeta(z)=\int_{0}^{z} \sqrt{a(\xi)} d \xi$. A renormalized entropy solution of (1.5) is a function $u \in L^{\infty}\left(0, T ; L^{1}\left(\mathbf{R}^{d}\right)\right)$ such that (i) $\nabla \zeta\left(T_{l}(u)\right)$ is square-integrable on $(0, T) \times \mathbf{R}^{d}$ for any $l>0$; (ii) for any convex $C^{2}$ entropy-entropy flux triple $(\eta, q, r)$, with $\eta^{\prime}$ bounded and $q^{\prime}=\eta^{\prime} f^{\prime}, r^{\prime}=\eta^{\prime} a$, there exists for any $l>0$ a nonnegative bounded Radon measure $\mu_{l}$ on $(0, T) \times \mathbf{R}^{d}$, whose total mass tends to zero as $l \uparrow \infty$, such that

$$
\begin{aligned}
& \partial_{t} \eta\left(T_{l}(u)\right)+\operatorname{div} q\left(T_{l}(u)\right)-\Delta r\left(T_{l}(u)\right) \\
& \quad \leq-\eta^{\prime \prime}\left(T_{l}(u)\right)\left|\nabla \zeta\left(T_{l}(u)\right)\right|^{2}+\mu_{l}(t, x) \quad \text { in } \mathcal{D}^{\prime}\left((0, T) \times \mathbf{R}^{d}\right) .
\end{aligned}
$$

Roughly speaking, (1.6) expresses the entropy condition satisfied by the truncated function $T_{l}(u)$. Of course, if $u$ is bounded by $M$, choosing $l>M$ in (1.6) yields the usual entropy formulation for $u$. In other words, a bounded renormalized entropy solution is an entropy solution. However, in contrast to the usual entropy formulation, (1.6) makes sense also when $u$ is merely $L^{1}$ and possibly unbounded. Intuitively the measure $\mu_{l}$ should be supported on the set $\{|u|=l\}$ and in particular carry information about the behavior of the "energy" on the set where $|u|$ is large. The requirement is that the energy should be small for large values of $|u|$, that is, the total mass of the renormalization measure $\mu_{l}$ should vanish as $l \uparrow \infty$. This is essential for proving uniqueness of a renormalized entropy solution. Being explicit, the existence proof reveals that $\mu_{l}\left((0, T) \times \mathbf{R}^{d}\right) \leq \int_{\left\{\left|u_{0}\right|>l\right\}}\left|u_{0}\right| d x \rightarrow 0$ as $l \uparrow \infty$.

We prove existence of a renormalized entropy solution to (1.1) using an approximation procedure based on artificial viscosity [42] and bounded data. We derive a priori estimates and pass to the limit in the approximations. 
Uniqueness of renormalized entropy solutions is proved by adapting the doubling of variables device due to Kružkov [32]. In the first order case, the uniqueness proof of Kružkov depends crucially on the fact that

$$
\nabla_{x} \Phi(x-y)+\nabla_{y} \Phi(x-y)=0, \quad \Phi \text { smooth function on } \mathbf{R}^{d},
$$

which allows for a cancellation of certain singular terms. The proof herein for the second order case relies in addition crucially on the following identity involving the Hessian matrices of $\Phi(x-y)$ :

$$
\nabla_{x x} \Phi(x-y)+2 \nabla_{x y} \Phi(x-y)+\nabla_{y y} \Phi(x-y)=0,
$$

which, when used together with the parabolic dissipation terms (like the one found in (1.6)), allows for a cancellation of certain singular terms involving the second order operator in (1.1). Compared to [19], our uniqueness proof is new even in the case of bounded entropy solutions.

The remaining part of this paper is organized as follows: In Section 2 we introduce the notion of a renormalized entropy solution for (1.1) and state our main well posedness theorem. The proof of this theorem is given in Section 3 (uniqueness) and Section 4 (existence).

\section{Definitions and Statement of main RESult}

We start by defining an entropy-entropy flux triple.

Definition 2.1 (entropy-entropy flux triple). For any convex $C^{2}$ entropy function $\eta: \mathbf{R} \rightarrow \mathbf{R}$ the corresponding entropy fluxes

$$
q=\left(q_{1}, \ldots, q_{d}\right): \mathbf{R} \rightarrow \mathbf{R}^{d} \quad \text { and } \quad r=\left(r_{i j}\right): \mathbf{R} \rightarrow \mathbf{R}^{d \times d}
$$

are defined by

$$
q^{\prime}(u)=\eta^{\prime}(u) f^{\prime}(u), \quad r^{\prime}(u)=\eta^{\prime}(u) a(u) .
$$

We will refer to $(\eta, q, R)$ as an entropy-entropy flux triple.

For $k=1, \ldots, K$ and $i=1, \ldots, d$, we let

$$
\zeta_{i k}(u)=\int_{0}^{u} \sigma_{i k}(\xi) d \xi, \quad \zeta_{k}(u)=\left(\zeta_{1 k}(u), \ldots, \zeta_{d k}(u)\right),
$$

and for any $\psi \in C(\mathbf{R})$

$$
\zeta_{i k}^{\psi}(u)=\int_{0}^{u} \psi(\xi) \sigma_{i k}(\xi) d \xi, \quad \zeta_{k}^{\psi}(u)=\left(\zeta_{1 k}^{\psi}(u), \ldots, \zeta_{d k}^{\psi}(u)\right) .
$$

Let us introduce the following set of vector fields:

$$
\begin{aligned}
& L^{2}\left(0, T ; L^{2}\left(\operatorname{div} ; \mathbf{R}^{d}\right)\right) \\
& \quad=\left\{w=\left(w_{1}, \ldots, w_{d}\right) \in\left(L^{2}\left((0, T) \times \mathbf{R}^{d}\right)\right)^{d}: \operatorname{div} w \in L^{2}\left((0, T) \times \mathbf{R}^{d}\right)\right\} .
\end{aligned}
$$

Following [19] we define an entropy solution as follows:

Definition 2.2 (entropy solution). An entropy solution of (1.1) is a measurable function $u:(0, T) \times \mathbf{R}^{d} \rightarrow \mathbf{R}$ satisfying the following conditions: 
(D.1) $u \in L^{\infty}\left(0, T ; L^{1}\left(\mathbf{R}^{d}\right)\right) \cap L^{\infty}\left((0, T) \times \mathbf{R}^{d}\right)$.

(D.2) For any $k=1, \ldots, K$,

$$
\zeta_{k}(u) \in L^{2}\left(0, T ; L^{2}\left(\operatorname{div} ; \mathbf{R}^{d}\right)\right) .
$$

(D.3) (chain rule) For any $k=1, \ldots, K$ and $\psi \in C(\mathbf{R})$,

$$
\operatorname{div} \zeta_{k}^{\psi}(u)=\psi(u) \operatorname{div} \zeta_{k}(u)
$$

a.e. in $(0, T) \times \mathbf{R}^{d}$ and in $L^{2}\left((0, T) \times \mathbf{R}^{d}\right)$.

(D.4) Define the parabolic dissipation measure $n_{l}^{u, \psi}(t, x)$ by

$$
n^{u, \psi}(t, x)=\psi(u(t, x)) \sum_{k=1}^{K}\left(\operatorname{div} \zeta_{k}(u(t, x))\right)^{2} .
$$

For any entropy-entropy flux triple $(\eta, q, r)$,

$$
\begin{aligned}
\partial_{t} \eta(u) & +\sum_{i=1}^{d} \partial_{x_{i}} q_{i}(u)-\sum_{i, j=1}^{d} \partial_{x_{i} x_{j}}^{2} r_{i j}(u) \\
& -\eta^{\prime}(u) F \leq-n^{u, \eta^{\prime \prime}} \text { in } \mathcal{D}^{\prime}\left((0, T) \times \mathbf{R}^{d}\right),
\end{aligned}
$$

that is, for any $0 \leq \phi \in \mathcal{D}\left((0, T) \times \mathbf{R}^{d}\right)$,

$$
\begin{gathered}
\int_{(0, T) \times \mathbf{R}^{d}}\left(\eta(u) \partial_{t} \phi+\sum_{i=1}^{d} q_{i}(u) \partial_{x_{i}} \phi+\sum_{i, j=1}^{d} r_{i j}(u) \partial_{x_{i} x_{j}}^{2} \phi\right) d x d t \\
+\int_{(0, T) \times \mathbf{R}^{d}} \eta^{\prime}(u) F \phi d x d t \geq \int_{(0, T) \times \mathbf{R}^{d}} n^{u, \eta^{\prime \prime}}(t, x) \phi d x d t .
\end{gathered}
$$

(D.5) The initial condition is taken in the following strong $L^{1}$ sense:

$$
\text { ess } \lim _{t \downarrow 0}\left\|u(\cdot, t)-u_{0}\right\|_{L^{1}\left(\mathbf{R}^{d}\right)}=0 \text {. }
$$

Note that, thanks to $(\mathbf{D} .1)$ and $\zeta_{k}(0)=0$ for $k=1, \ldots, d$, condition $(\mathbf{D} .2)$ is satisfied once we know that

$$
\operatorname{div} \zeta_{k}(u)=\sum_{i=1}^{d} \partial_{x_{i}} \zeta_{i k}(u) \in L^{2}\left((0, T) \times \mathbf{R}^{d}\right) .
$$

An important contribution of Chen and Perthame [19] is to make explicit the point that the chain rule (D.3) should be included in the definition of an entropy solution in the anisotropic diffusion case. They also note that (D.3) is automatically fulfilled when $a(u)$ is a diagonal matrix, and can then be deleted from Definition 2.2. This remark applies to the isotropic case (1.5).

Uniqueness of an entropy solution in the sense of Definition 2.2 was proved in [19] using a kinetic formulation and regularization by convolution. 
The present paper offers an alternative proof based on the more classical Kružkov method of doubling the variables [32].

Let us mention that (D.4) implies the following Kružkov type entropy condition for all $c \in \mathbf{R}$ :

$$
\begin{aligned}
\partial_{t}|u-c| & +\sum_{i=1}^{d} \partial_{x_{i}}\left[\operatorname{sign}(u-c)\left(f_{i}(u)-f_{i}(c)\right)\right] \\
& -\sum_{i, j=1}^{d} \partial_{x_{i} x_{j}}^{2}\left[\operatorname{sign}(u-c)\left(A_{i j}(u)-A_{i j}(c)\right)\right] \\
& -\operatorname{sign}(u-c) F \leq 0, \quad A_{i j}^{\prime}(\cdot)=a_{i j}(\cdot) .
\end{aligned}
$$

In the isotropic case (1.5), (2.2) simplifies to

$$
\begin{aligned}
& \partial_{t}|u-c|+\operatorname{div}[\operatorname{sign}(u-c)(f(u)-f(c))] \\
&-\Delta|A(u)-A(c)|-\operatorname{sign}(u-c) F \leq 0, \quad \forall c \in \mathbf{R} .
\end{aligned}
$$

After Carrillo's work [15, 14], it is known that (2.3) implies uniqueness in the isotropic case (1.5). In the anisotropic case (1.1), (2.2) is not sufficient for uniqueness. Indeed, it is necessary to explicitly include the parabolic dissipation measure in the entropy condition, as is done in (D.4).

As we discussed in Section 1, for unbounded $L^{1}$ solutions $u$ Definition 2.2 is in general not meaningful. In [19] the authors use a notion of kinetic solutions to handle unbounded $L^{1}$ solutions. It is the purpose of this paper to use instead a notion renormalized entropy solutions.

Before we can introduce this notion, let us recall the definition of the Lipschitz continuous truncation function $T_{l}: \mathbf{R} \rightarrow \mathbf{R}$ at height $l>0$, which is defined by

$$
T_{l}(u)= \begin{cases}-l, & \text { if } u<-l, \\ u, & \text { if }|u| \leq l, \\ l, & \text { if } u>l .\end{cases}
$$

We then suggest the following notion of an $L^{1}$ solution:

Definition 2.3 (renormalized entropy solution). A renormalized entropy solution of (1.1) is a measurable function $u:(0, T) \times \mathbf{R}^{d} \rightarrow \mathbf{R}$ satisfying the following conditions:

(D.1) $u \in L^{\infty}\left(0, T ; L^{1}\left(\mathbf{R}^{d}\right)\right)$.

(D.2) For any $k=1, \ldots, K$,

$$
\zeta_{k}\left(T_{l}(u)\right) \in L^{2}\left(0, T ; L^{2}\left(\operatorname{div} ; \mathbf{R}^{d}\right)\right), \quad \forall l>0 .
$$

(D.3) (renormalized chain rule) For any $k=1, \ldots, K$ and $\psi \in C(\mathbf{R})$,

$$
\operatorname{div} \zeta_{k}^{\psi}\left(T_{l}(u)\right)=\psi\left(T_{l}(u)\right) \operatorname{div} \zeta_{k}\left(T_{l}(u)\right) \quad \forall l>0,
$$


a.e. in $(0, T) \times \mathbf{R}^{d}$ and in $L^{2}\left((0, T) \times \mathbf{R}^{d}\right)$.

(D.4) For any $l>0$, define the renormalized parabolic dissipation measure $n_{l}^{u, \psi}(t, x)$ by

$$
n_{l}^{u, \psi}(t, x)=\psi\left(T_{l}(u(t, x))\right) \sum_{k=1}^{K}\left(\operatorname{div} \zeta_{k}\left(T_{l}(u(t, x))\right)\right)^{2} .
$$

For any $l>0$ and any entropy-entropy flux triple $(\eta, q, r)$, with $\left|\eta^{\prime}\right|$ bounded by $K$ (for some given $K$ ), there exists a nonnegative bounded Radon measure $\mu_{l}^{u, K}$ on $(0, T) \times \mathbf{R}^{d}$ such that

$$
\begin{aligned}
\partial_{t} \eta\left(T_{l}(u)\right) & +\sum_{i=1}^{d} \partial_{x_{i}} q_{i}\left(T_{l}(u)\right)-\sum_{i, j=1}^{d} \partial_{x_{i} x_{j}}^{2} r_{i j}^{\prime}\left(T_{l}(u)\right) \\
& -\eta^{\prime}\left(T_{l}(u)\right) F \leq-n_{l}^{u, \eta^{\prime \prime}}+\mu_{l}^{u, K} \quad \text { in } \mathcal{D}^{\prime}\left((0, T) \times \mathbf{R}^{d}\right),
\end{aligned}
$$

that is, for any $0 \leq \phi \in \mathcal{D}\left((0, T) \times \mathbf{R}^{d}\right)$,

$$
\begin{gathered}
\int_{(0, T) \times \mathbf{R}^{d}}\left(\eta\left(T_{l}(u)\right) \partial_{t} \phi+\sum_{i=1}^{d} q_{i}\left(T_{l}(u)\right) \partial_{x_{i}} \phi+\sum_{i, j=1}^{d} r_{i j}\left(T_{l}(u)\right) \partial_{x_{i} x_{j}}^{2} \phi\right) d x d t \\
+\int_{(0, T) \times \mathbf{R}^{d}} \eta^{\prime}\left(T_{l}(u)\right) F(t, x) \phi \\
\quad \geq \int_{(0, T) \times \mathbf{R}^{d}} n_{l}^{u, \eta^{\prime \prime}}(t, x) \phi d x d t-\int_{(0, T) \times \mathbf{R}^{d}} \phi d \mu_{l}^{u, K}(t, x) .
\end{gathered}
$$

(D.5) The total mass of the renormalization measure $\mu_{l}^{u, K}$ vanishes as $l \uparrow \infty$, that is,

$$
\lim _{l \uparrow \infty} \mu_{l}^{u, K}\left((0, T) \times \mathbf{R}^{d}\right)=0 .
$$

(D.6) The initial condition is taken in the following strong $L^{1}$ sense:

$$
\underset{t \downarrow 0}{\operatorname{ess} \lim }\left\|u(\cdot, t)-u_{0}\right\|_{L^{1}\left(\mathbf{R}^{d}\right)}=0 \text {. }
$$

Note that since $T_{l}(u) \in L^{\infty}\left((0, T) \times \mathbf{R}^{d}\right)$, the integrals in (2.5) are all well defined. Moreover, if a renormalized entropy solution $u$ belongs to $L^{\infty}\left((0, T) \times \mathbf{R}^{d}\right)$, then it is also an entropy solution in the sense Definition 2.2 (simply let $l \uparrow \infty$ in Definition 2.3).

Our main well posedness result is contained in the following theorem, which is proved in Sections 3 (uniqueness) and 4 (existence).

Theorem 2.1 (well posedness). Suppose (1.2), (1.3), and (1.4) hold. Then there exists a unique renormalized entropy solution $u$ of (1.1). 
In applications [13] it may be of interest to require less regularity of $\sigma(\cdot)$ than allowed for by (1.3). Although we do not pursue this here (but see Lemmas 2.1 and 2.2 below), it is possible to prove Theorem 2.1 under the assumption that $\sigma(\cdot)$ is piecewise smooth.

In the isotropic case it is not necessary to include the chain rule (D.3) as a part of Definition 2.3 since it is then automatically fulfilled, as the following two lemmas show.

Lemma 2.1 (chain rule). Let $0 \leq \sigma \in L_{\mathrm{loc}}^{\infty}(\mathbf{R})$ and $\psi \in L_{\mathrm{loc}}^{\infty}(\mathbf{R})$. Set

$$
\beta(z)=\int_{0}^{z} \sigma(\xi) d \xi, \quad \beta^{\psi}(z)=\int_{0}^{z} \psi(\xi) \sigma(\xi) d \xi .
$$

Then, for any measurable function $u$ such that $\beta\left(T_{l}(u)\right) \in H_{\mathrm{loc}}^{1}\left(\mathbf{R}^{d}\right)$,

$$
\nabla \beta^{\psi}\left(T_{l}(u(x))\right)=\psi\left(T_{l}(u(x))\right) \nabla \beta\left(T_{l}(u(x))\right), \quad \forall l>0,
$$

for a.e. $x \in \mathbf{R}^{d}$ and in $L_{\mathrm{loc}}^{2}\left(\mathbf{R}^{d}\right)$.

Proof. As in [19], (2.6) is a consequence of standard Sobolev space theory (see, e.g., [28]). For the sake of completeness, let us sketch a proof.

Define the lower semicontinuous function $\beta^{-1}: \mathbf{R} \rightarrow \mathbf{R}$ by

$$
\beta^{-1}(w):=\inf \{\xi \in \mathbf{R} \mid \beta(\xi)=w\} .
$$

Denote by $E \subset \mathbf{R}$ the set

$$
E=\left\{w \in \mathbf{R} \text { such that } \beta^{-1}(\cdot) \text { is discontinuous at } w\right\} .
$$

Since $\beta(\cdot)$ is nondecreasing, $E$ is at most countable. Introduce the function

$$
v:=\beta\left(T_{l}(u)\right) \in H_{\mathrm{loc}}^{1}\left(\mathbf{R}^{d}\right) \subset W_{\mathrm{loc}}^{1,1}\left(\mathbf{R}^{d}\right)
$$

and the corresponding set

$$
\mathcal{E}=\left\{x \in \mathbf{R}^{d}: v(x) \in E\right\} \subset \mathbf{R}^{d} .
$$

Then the classical chain rule from Sobolev space theory gives

$$
\partial_{x_{i}} \Psi(v(x))=\Psi^{\prime}(v(x)) \partial_{x_{i}} v(x), \quad \text { for a.e. } x \in \mathbf{R}^{d} \backslash \mathcal{E}, i=1, \ldots, d,
$$

where $\Psi: \mathbf{R} \rightarrow \mathbf{R}$ is defined by

$$
\Psi^{\prime}(v)=\psi\left(\beta^{-1}(v)\right), \quad v \in \mathbf{R} .
$$

However, on $\mathcal{E}$ the $W_{\text {loc }}^{1,1}$ function $v=v(x)$ is constant and hence from standard Sobolev space theory

$$
\partial_{x_{i}} \Psi(v)=\partial_{x_{i}} v=0, \quad \text { for a.e. } x \in \mathcal{E}, i=1, \ldots, d .
$$

By the definition of $v$ and since $\beta^{-1}(\beta(\xi))=\xi$ for all $\xi$, we obtain (2.6).

Lemma 2.2. Let $u$ be a renormalized entropy solution to (1.1) with a(u) being a diagonal matrix:

$$
a(u)=\operatorname{diag}\left(a_{1}(u), \ldots, a_{d}(u)\right), \quad 0 \leq a_{i} \in L_{\mathrm{loc}}^{\infty}(\mathbf{R}), \quad i=1, \ldots, d .
$$


For any $\psi \in L_{\mathrm{loc}}^{\infty}(\mathbf{R})$ and $i=1, \ldots, d$, let

$$
\zeta_{i}(u)=\int_{0}^{u} \sqrt{a_{i}(\xi)} d \xi, \quad \zeta_{i}^{\psi}(u)=\int_{0}^{u} \psi(\xi) \sqrt{a_{i}(\xi)} d \xi .
$$

Then for $i=1, \ldots, d$

$$
\partial_{x_{i}} \zeta^{\psi_{i}}\left(T_{l}(u(x, t))\right)=\psi\left(T_{l}(u(x, t))\right) \partial_{x_{i}} \zeta_{i}\left(T_{l}(u(x, t))\right), \quad \forall l>0,
$$

for a.e. $(x, t) \in(0, T) \times \mathbf{R}^{d}$ and in $L^{2}\left((0, T) \times \mathbf{R}^{d}\right)$.

Proof. Since $\partial_{x_{i}} \zeta_{i}\left(T_{l}(u)\right) \in L^{2}\left((0, T) \times \mathbf{R}^{d}\right)$ and $u \in L^{\infty}\left(0, T ; L^{1}\left(\mathbf{R}^{d}\right)\right)$ (see Definition 2.3), we have

$$
\zeta_{i}\left(T_{l}(u)\right) \in L^{2}\left(0, T ; H^{1}\left(\mathbf{R}^{d}\right)\right) .
$$

Hence $\zeta_{i}\left(T_{l}(u(\cdot, t))\right) \in H^{1}\left(\mathbf{R}^{d}\right)$ for a.e. $t \in(0, T)$. Then $(2.7)$ follows from an application of Lemma 2.1.

\section{UNIQUENESS OF RENORMALIZED ENTROPY SOLUTION}

For the uniqueness proof, we need a $C^{1}$ approximation of $\operatorname{sign}(\cdot)$ and a corresponding $C^{2}$ approximation of the Kružkov entropy flux $|\cdot-c|, c \in \mathbf{R}$.

Lemma 3.1. For $\varepsilon>0$, set

$$
\operatorname{sign}_{\varepsilon}(\xi)= \begin{cases}-1, & \xi<\varepsilon, \\ \sin \left(\frac{\pi}{2 \varepsilon} \xi\right), & |\xi| \leq \varepsilon, \\ 1, & \xi>\varepsilon\end{cases}
$$

For each $c \in \mathbf{R}$, the corresponding entropy function

$$
u \mapsto \eta_{\varepsilon}(u, c)=\int_{c}^{u} \operatorname{sign}_{\varepsilon}(\xi) d \xi
$$

is convex and belongs to $C^{2}(\mathbf{R})$ with $\eta_{\varepsilon}^{\prime \prime} \in C_{c}(\mathbf{R})$ and $\left|\eta_{\varepsilon}^{\prime}\right| \leq 1$ (so that the constant $K$ appearing in Definition 2.3 is 1$)$. Moreover, $\eta_{\varepsilon}$ is symmetric in the sense that $\eta_{\varepsilon}(z, c)=\eta_{\varepsilon}(c, z)$ and for all $u \in \mathbf{R}$

$$
\eta_{\varepsilon}(u, c) \rightarrow \eta(u, c):=|u-c| \quad \text { as } \varepsilon \downarrow 0 .
$$

For each $c \in \mathbf{R}$ and $1 \leq i, j \leq d$, we define the entropy flux functions

$$
\begin{aligned}
& u \mapsto q_{i}^{\varepsilon}(u, c)=\int_{c}^{u} \operatorname{sign}_{\varepsilon}(\xi-c) f_{i}^{\prime}(\xi) d \xi, \\
& u \mapsto r_{i j}^{\varepsilon}(u, c)=\int_{c}^{u} \operatorname{sign}_{\varepsilon}(\xi-c) A_{i j}^{\prime}(\xi) d \xi,
\end{aligned}
$$

where $A_{i j}^{\prime}(\cdot)=a_{i j}(\cdot)$ for $1 \leq i, j \leq d$. Then as $\varepsilon \downarrow 0$

$$
\begin{aligned}
& q_{i}^{\varepsilon}(u, c) \rightarrow \eta(u, c):=\operatorname{sign}(u-c)(f(u)-f(c)), \\
& r_{i j}^{\varepsilon}(\cdot, c) \rightarrow r_{i j}(u, c):=\operatorname{sign}(u-c)\left(A_{i j}(u)-A_{i j}(c)\right),
\end{aligned}
$$

for $1 \leq i, j \leq d$. Let $q^{\varepsilon}=\left(q_{1}^{\varepsilon}, \ldots, q_{d}^{\varepsilon}\right), r^{\varepsilon}=\left(r_{i j}^{\varepsilon}\right)$, and similarly for $q, r$. 
The function (3.1) is taken from [17]. The Kružkov entropy fluxes $q, r$ in (3.3) are symmetric. This is, however, not true for the entropy fluxes $q^{\varepsilon}, r^{\varepsilon}$, but the symmetry error goes to zero as $\varepsilon \downarrow 0$, that is,

$$
q^{\varepsilon}(u, c)-q^{\varepsilon}(c, u) \rightarrow 0, \quad r^{\varepsilon}(u, c)-r^{\varepsilon}(c, u) \rightarrow 0,
$$

for all $u, c \in \mathbf{R}$, and this is sufficient for proving uniqueness.

We are now ready to prove uniqueness of renormalized entropy solutions.

Theorem 3.1 (uniqueness). Suppose (1.3) and (1.4) hold. Let $u$ and $v$ be renormalized entropy solutions of (1.1) with data $F \in L^{1}\left((0, T) \times \mathbf{R}^{d}\right)$, $u_{0} \in L^{1}\left(\mathbf{R}^{d}\right)$ and $G \in L^{1}\left((0, T) \times \mathbf{R}^{d}\right), v_{0} \in L^{1}\left(\mathbf{R}^{d}\right)$, respectively. Then for a.e. $t \in(0, T)$,

$$
\begin{aligned}
& \|u(\cdot, t)-v(\cdot, t)\|_{L^{1}\left(\mathbf{R}^{d}\right)} \\
& \quad \leq\left\|u_{0}-v_{0}\right\|_{L^{1}\left(\mathbf{R}^{d}\right)}+\int_{0}^{t}\|F(s, \cdot)-G(s, \cdot)\|_{L^{1}\left(\mathbf{R}^{d}\right)} d s .
\end{aligned}
$$

In particular, the Cauchy problem (1.1) admits at most one renormalized entropy solution.

Proof. We shall prove (3.4) using Kružkov's doubling of variables method [32]. When it is notationally convenient we drop the domain of integration.

Let $\left(\eta_{\varepsilon}, q_{i}^{\varepsilon}, r_{i j}^{\varepsilon}\right)$ be the entropy flux triple defined in Lemma 3.1, and denote by $\mu_{l}^{u}, \mu_{l}^{v}$ the corresponding renormalization measures.

From the definition of an renormalized entropy solution for $u=u(t, x)$,

$$
\begin{gathered}
\int\left(\eta_{\varepsilon}\left(T_{l}(u), c\right) \partial_{t} \phi+\sum_{i=1}^{d} q_{i}^{\varepsilon}\left(T_{l}(u), c\right) \partial_{x_{i}} \phi+\sum_{i, j=1}^{d} r_{i j}^{\varepsilon}\left(T_{l}(u), c\right) \partial_{x_{i} x_{j}}^{2} \phi\right) d x d t \\
-\int \operatorname{sign}_{\varepsilon}\left(T_{l}(u)-c\right) F(t, x) \phi d x d t \\
\geq \int n^{u, \operatorname{sign}_{\varepsilon}^{\prime}(-c)}(t, x) \phi d x d t-\int \phi d \mu_{l}^{u}(t, x),
\end{gathered}
$$

$\forall c \in \mathbf{R}, \forall l>0$ and for $0 \leq \phi=\phi(t, x) \in \mathcal{D}\left((0, T) \times \mathbf{R}^{d}\right)$.

From the definition of renormalized entropy solution for $u=u(s, y)$,

$$
\begin{gathered}
\int\left(\eta_{\varepsilon}\left(T_{l}(v), c\right) \partial_{s} \phi+\sum_{i=1}^{d} q_{i}^{\varepsilon}\left(T_{l}(v), c\right) \partial_{y_{j}} \phi+\sum_{i, j=1}^{d} r_{i j}^{\varepsilon}\left(T_{l}(v), c\right) \partial_{y_{i} y_{j}}^{2} \phi\right) d y d s \\
-\int \operatorname{sign}_{\varepsilon}\left(T_{l}(v)-c\right) G(s, y) \phi d y d s \\
\geq \int n^{v, \operatorname{sign}_{\varepsilon}^{\prime}(c-\cdot)}(s, y) \phi d y d s-\int \phi d \mu_{l}^{v}(s, y),
\end{gathered}
$$

$\forall c \in \mathbf{R}, \forall l>0$ and for every $0 \leq \phi=\phi(s, y) \in \mathcal{D}\left((0, T) \times \mathbf{R}^{d}\right)$. 
Choose $c=T_{l}(v(s, y))$ in (3.5) and integrate over $(s, y)$. Choose $c=$ $T_{l}(u(t, x))$ in $(3.6)$ and integrate over $(t, x)$. Then adding the two resulting inequalities yields

$$
\begin{gathered}
\int\left(\eta_{\varepsilon}\left(T_{l}(u), T_{l}(v)\right)\left(\partial_{t}+\partial_{s}\right) \phi\right. \\
+\sum_{i=1}^{d}\left[q_{i}^{\varepsilon}\left(T_{l}(u), T_{l}(v)\right) \partial_{x_{i}} \phi+q_{i}^{\varepsilon}\left(T_{l}(v), T_{l}(u)\right) \partial_{y_{i}} \phi\right] \\
\left.+\sum_{i, j=1}^{d}\left[r_{i j}^{\varepsilon}\left(T_{l}(u), T_{l}(v)\right) \partial_{x_{i} x_{j}}^{2} \phi+r_{i j}^{\varepsilon}\left(T_{l}(v), T_{l}(u)\right) \partial_{y_{i} y_{j}}^{2} \phi\right]\right) d x d t d y d s \\
-\int \operatorname{sign}_{\varepsilon}\left(T_{l}(u)-T_{l}(v)\right)(F(t, x)-G(s, y)) d x d t d y d s \\
\geq \int\left(n^{u, \operatorname{sign}_{\varepsilon}^{\prime}(\cdot-c)}(t, x)+n^{v, \operatorname{sign}_{\varepsilon}^{\prime}(\cdot-c)}(s, y)\right) \phi d x d t d y d s \\
-\int \phi(t, x, s, y) d \mu_{l}^{u}(t, x) d y d s \\
-\int \phi(t, x, s, y) d \mu_{l}^{v}(s, y) d x d t
\end{gathered}
$$

where $\phi=\phi(t, x, s, y)$ is any nonnegative function in $\mathcal{D}\left(\left((0, T) \times \mathbf{R}^{d}\right)^{2}\right)$.

We introduce next a function $0 \leq \omega \in \mathcal{D}(\mathbf{R})$ that satisfies $\omega(\sigma)=\omega(-\sigma)$, $\omega(\sigma)=0$ for $|\sigma| \geq 1$, and $\int_{\mathbf{R}} \omega(\sigma) d \sigma=1$. For $\rho>0$ and $z \in \mathbf{R}$, let $\omega_{\rho}(z)=\frac{1}{\rho} \omega\left(\frac{z}{\rho}\right)$. We take our test function $\phi=\phi(t, x, s, y)$ to be of the form

$$
\phi(t, x, s, y)=\varphi\left(\frac{t+s}{2}, \frac{x+y}{2}\right) \omega_{\rho}\left(\frac{t-s}{2}, \frac{x-y}{2}\right),
$$

where $\varphi \in \mathcal{D}\left((0, T) \times \mathbf{R}^{d}\right), 0 \leq \varphi \leq 1$, and $\omega_{\rho}\left(\frac{t-s}{2}, \frac{x-y}{2}\right)=\omega_{\rho}\left(\frac{x-y}{2}\right) \omega_{\rho}\left(\frac{t-s}{2}\right)$.

With this choice, we have

$$
\left(\partial_{t}+\partial_{s}\right) \phi=\left(\partial_{t}+\partial_{s}\right) \varphi\left(\frac{t+s}{2}, \frac{x+y}{2}\right) \omega_{\rho}\left(\frac{t-s}{2}, \frac{x-y}{2}\right)
$$

and

$$
\left(\nabla_{x}+\nabla_{y}\right) \phi=\left(\nabla_{x}+\nabla_{y}\right) \varphi\left(\frac{t+s}{2}, \frac{x+y}{2}\right) \omega_{\rho}\left(\frac{t-s}{2}, \frac{x-y}{2}\right) .
$$

Introduce the Hessian matrices

$$
\nabla_{x x} \phi=\left(\partial_{x_{i} x_{j}}^{2} \phi\right), \quad \nabla_{x y} \phi=\left(\partial_{x_{i} y_{j}}^{2} \phi\right), \quad \nabla_{y y} \phi=\left(\partial_{y_{i} y_{j}}^{2} \phi\right) .
$$

Then one can check that the following crucial matrix equality holds:

$$
\left(\nabla_{x x}+2 \nabla_{x y}+\nabla_{y y}\right) \phi=\left(\nabla_{x x}+2 \nabla_{x y}+\nabla_{y y}\right) \varphi\left(\frac{t+s}{2}, \frac{x+y}{2}\right) \omega_{\rho}\left(\frac{t-s}{2}, \frac{x-y}{2}\right) .
$$


Note that the two latter properties imply that for $1 \leq i \leq d$

$$
\begin{aligned}
& q_{i}^{\varepsilon}\left(T_{l}(u), T_{l}(v)\right) \partial_{x_{i}} \phi+q_{i}^{\varepsilon}\left(T_{l}(v), T_{l}(u)\right) \partial_{y_{i}} \phi \\
& =q_{i}^{\varepsilon}\left(T_{l}(u), T_{l}(v)\right)\left(\partial_{x_{i}}+\partial_{y_{i}}\right) \varphi\left(\frac{t+s}{2}, \frac{x+y}{2}\right) \omega_{\rho}\left(\frac{t-s}{2}, \frac{x-y}{2}\right) \\
& \quad+\left[q_{i}^{\varepsilon}\left(T_{l}(v), T_{l}(u)\right)-q_{i}^{\varepsilon}\left(T_{l}(u), T_{l}(v)\right)\right] \partial_{y_{i}} \phi
\end{aligned}
$$

and for $1 \leq i, j \leq d$

$$
\begin{aligned}
& r_{i j}^{\varepsilon}\left(T_{l}(u), T_{l}(v)\right) \partial_{x_{i} x_{j}}^{2} \phi+r_{i j}^{\varepsilon}\left(T_{l}(v), T_{l}(u)\right) \partial_{y_{i} y_{j}}^{2} \phi \\
& =r_{i j}^{\varepsilon}\left(T_{l}(u), T_{l}(v)\right)\left(\partial_{x_{i} x_{j}}^{2}+2 \partial_{x_{i} y_{j}}^{2}+\partial_{y_{i} y_{j}}^{2}\right) \varphi\left(\frac{t+s}{2}, \frac{x+y}{2}\right) \omega_{\rho}\left(\frac{t-s}{2}, \frac{x-y}{2}\right) \\
& \quad-2 r_{i j}^{\varepsilon}\left(T_{l}(u), T_{l}(v)\right) \partial_{x_{i} y_{j}}^{2} \phi \\
& \quad+\left[r_{i j}^{\varepsilon}\left(T_{l}(v), T_{l}(u)\right)-r_{i j}^{\varepsilon}\left(T_{l}(u), T_{l}(v)\right)\right] \partial_{y_{i} y_{j}}^{2} \phi .
\end{aligned}
$$

We also have

$$
\begin{aligned}
& -\int \phi(t, x, s, y) d \mu_{l}^{u}(t, x) d y d s \\
& \quad \geq-\int \omega_{\rho}\left(\frac{t-s}{2}, \frac{x-y}{2}\right) d y d s d \mu_{l}^{u}(t, x) \geq-\mu_{l}^{u}\left((0, T) \times \mathbf{R}^{d}\right)
\end{aligned}
$$

and similarly

$$
-\int \phi(t, x, s, y) d \mu_{l}^{v}(s, y) d x d t \geq-\mu_{l}^{v}\left((0, T) \times \mathbf{R}^{d}\right) .
$$

Insertion of (3.8)-(3.12) into (3.7) gives

$$
\begin{gathered}
\int\left(\eta_{\varepsilon}\left(T_{l}(u), T_{l}(v)\right)\left(\partial_{t}+\partial_{s}\right) \varphi\left(\frac{t+s}{2}, \frac{x+y}{2}\right)\right. \\
+\sum_{i=1}^{d} q_{i}^{\varepsilon}\left(T_{l}(u), T_{l}(v)\right)\left(\partial_{x_{i}}+\partial_{y_{i}}\right) \varphi\left(\frac{t+s}{2}, \frac{x+y}{2}\right) \\
\left.+\sum_{i, j=1}^{d} r_{i j}^{\varepsilon}\left(T_{l}(u), T_{l}(v)\right)\left(\partial_{x_{i} x_{j}}^{2}+2 \partial_{x_{i} y_{j}}^{2}+\partial_{y_{i} y_{j}}^{2}\right) \varphi\left(\frac{t+s}{2}, \frac{x+y}{2}\right)\right) \\
\times \omega_{\rho}\left(\frac{t-s}{2}, \frac{x-y}{2}\right) d x d t d y d s \\
\quad-\int \operatorname{sign}_{\varepsilon}\left(T_{l}(u)-T_{l}(v)\right)(F(t, x)-G(s, y)) d x d t d y d s \\
\geq E_{1}(\varepsilon)+E_{2}(\varepsilon)+E_{3}(\varepsilon)-\mu_{l}^{v}\left((0, T) \times \mathbf{R}^{d}\right)-\mu_{l}^{v}\left((0, T) \times \mathbf{R}^{d}\right),
\end{gathered}
$$


where $E_{j}(\varepsilon)=\int_{\left((0, T) \times \mathbf{R}^{d}\right)^{2}} I_{j}(\varepsilon) d x d t d y d s, j=1,2,3$, with

$$
\begin{aligned}
I_{1}(\varepsilon) & =\left(n^{u, \operatorname{sign}_{\varepsilon}^{\prime}(\cdot-c)}(t, x)+n^{v, \operatorname{sign}_{\varepsilon}^{\prime}(\cdot-c)}(s, y)\right) \phi \\
I_{2}(\varepsilon) & =2 \sum_{i, j=1}^{d} r_{i j}^{\varepsilon}\left(T_{l}(u), T_{l}(v)\right) \partial_{x_{i} y_{j}}^{2} \phi \\
I_{3}(\varepsilon)=\sum_{i=1}^{d}\left[q_{i}^{\varepsilon}\left(T_{l}(u), T_{l}(v)\right)-q_{i}^{\varepsilon}\left(T_{l}(v), T_{l}(u)\right)\right] \partial_{y_{i}} \phi & \\
& \quad+\sum_{i, j=1}^{d}\left[r_{i j}^{\varepsilon}\left(T_{l}(u), T_{l}(v)\right)-r_{i j}^{\varepsilon}\left(T_{l}(v), T_{l}(u)\right)\right] \partial_{y_{i} y_{j}}^{2} \phi .
\end{aligned}
$$

Thanks to Lemma 3.1, $\lim _{\varepsilon \downarrow 0} E_{3}(\varepsilon)=0$ and

$$
\lim _{\varepsilon \downarrow 0} E_{2}(\varepsilon)=\int 2 \sum_{i, j=1}^{d} r_{i j}\left(T_{l}(u), T_{l}(v)\right) \partial_{x_{i} y_{j}}^{2} \phi d x d t d y d s .
$$

Our goal now is to show that

$$
\lim _{\varepsilon \downarrow 0} E_{1}(\varepsilon)+\lim _{\varepsilon \downarrow 0} E_{2}(\varepsilon) \geq 0
$$

To this end, note first that, since $\operatorname{sign}_{\varepsilon}^{\prime}(\cdot) \geq 0$,

$$
I_{1}(\varepsilon) \geq 2 \sum_{k=1}^{K} \operatorname{sign}_{\varepsilon}^{\prime}\left(T_{l}(u)-T_{l}(v)\right) \operatorname{div}_{x} \zeta_{k}\left(T_{l}(u)\right) \operatorname{div}_{y} \zeta_{k}\left(T_{l}(v)\right) \phi,
$$

so that

$$
\begin{array}{r}
E_{1}(\varepsilon) \geq \int 2 \sum_{k=1}^{K} \operatorname{sign}_{\varepsilon}^{\prime}\left(T_{l}(u)-T_{l}(v)\right) \operatorname{div}_{x} \zeta_{k}\left(T_{l}(u)\right) \\
\times \operatorname{div}_{y} \zeta_{k}\left(T_{l}(v)\right) \phi d x d t d y d s
\end{array}
$$

Invoking the chain rule (D.3) in Definition 2.3 (we can do this since $\left.\operatorname{sign}_{\varepsilon}^{\prime}(\cdot) \in C(\mathbf{R})\right)$, we have for $1 \leq k \leq K$

$$
\begin{aligned}
& \operatorname{sign}_{\varepsilon}^{\prime}\left(T_{l}(u)-T_{l}(v)\right) \operatorname{div}_{x} \zeta_{k}\left(T_{l}(u)\right)=\operatorname{div}_{x} \zeta_{k}^{\operatorname{sign}_{\varepsilon}^{\prime}\left(\cdot-T_{l}(v)\right)}\left(T_{l}(u)\right) \\
& \operatorname{sign}_{\varepsilon}^{\prime}\left(T_{l}(u)-T_{l}(v)\right) \operatorname{div}_{y} \zeta_{k}\left(T_{l}(v)\right)=\operatorname{div}_{y} \zeta_{k}^{\operatorname{sign}_{\varepsilon}^{\prime}\left(T_{l}(u)-\cdot\right)}\left(T_{l}(v)\right)
\end{aligned}
$$


If we now use (3.16), then we can continue as follows:

$$
\begin{aligned}
& E_{1} \geq \int \sum_{k=1}^{K} \operatorname{div}_{x} \zeta_{k}\left(T_{l}(u)\right) \operatorname{div}_{y} \zeta_{k}^{\operatorname{sign}_{\varepsilon}^{\prime}\left(T_{l}(u)-\cdot\right)}\left(T_{l}(v)\right) \phi d x d t d y d s \\
&+\int \sum_{k=1}^{K} \operatorname{div}_{x} \zeta_{k}^{\operatorname{sign}_{\varepsilon}^{\prime}\left(\cdot-T_{l}(v)\right)}\left(T_{l}(u)\right) \operatorname{div}_{y} \zeta_{k}\left(T_{l}(v)\right) \phi d x d t d y d s \\
&=- \int \sum_{k=1}^{K} \operatorname{div}_{x} \zeta_{k}\left(T_{l}(u)\right) \zeta_{k}^{\operatorname{sign}_{\varepsilon}^{\prime}\left(T_{l}(u)-\cdot\right)}\left(T_{l}(v)\right) \cdot \nabla_{y} \phi d x d t d y d s \\
&-\int \sum_{k=1}^{K} \zeta_{k}^{\operatorname{sign}_{\varepsilon}^{\prime}\left(\cdot-T_{l}(v)\right)}\left(T_{l}(u)\right) \cdot \nabla_{x} \phi \operatorname{div}_{y} \zeta_{k}\left(T_{l}(v)\right) d x d t d y d s \\
&=- \int \sum_{k=1}^{K} \sum_{j=1}^{d}\left(\int_{T_{l}(u)}^{T_{l}(v)} \operatorname{sign}_{\varepsilon}^{\prime}\left(T_{l}(u)-\xi\right) \sigma_{j k}(\xi) d \xi\right) \\
&-\int \sum_{k=1}^{K} \sum_{i=1}^{d}\left(\int_{T_{l}(v)}^{T_{l}(u)} \operatorname{sign}_{\varepsilon}^{\prime}\left(\xi-T_{l}(v)\right) \sigma_{i k}(\xi) d \xi\right) \\
& \times \operatorname{div}_{y} \zeta_{k}\left(T_{l}(v)\right) \partial_{x_{i}} \phi d x d t d y d s .
\end{aligned}
$$

We observe next that for $1 \leq k \leq K$ and $1 \leq i, j \leq d$

$$
\begin{aligned}
& \lim _{\varepsilon \downarrow 0} \int_{T_{l}(u)}^{T_{l}(v)} \operatorname{sign}_{\varepsilon}^{\prime}\left(T_{l}(u)-\xi\right) \sigma_{j k}(\xi) d \xi=-\operatorname{sign}\left(T_{l}(u)-T_{l}(v)\right) \sigma_{j k}\left(T_{l}(u)\right), \\
& \lim _{\varepsilon \downarrow 0} \int_{T_{l}(v)}^{T_{l}(u)} \operatorname{sign}_{\varepsilon}^{\prime}\left(\xi-T_{l}(v)\right) \sigma_{i k}(\xi) d \xi=\operatorname{sign}\left(T_{l}(u)-T_{l}(v)\right) \sigma_{i k}\left(T_{l}(v)\right) .
\end{aligned}
$$


Hence, by the dominated convergence theorem,

$$
\begin{aligned}
& \lim _{\varepsilon \downarrow 0} E_{1} \geq \int \sum_{k=1}^{K} \sum_{j=1}^{d} \operatorname{sign}(\left.T_{l}(u)-T_{l}(v)\right) \sigma_{j k}\left(T_{l}(u)\right) \\
& \times \operatorname{div}_{x} \zeta_{k}\left(T_{l}(u)\right) \partial_{y_{j}} \phi d x d t d y d s \\
&-\int \sum_{k=1}^{K} \sum_{i=1}^{d} \operatorname{sign}\left(T_{l}(u)-T_{l}(v)\right) \sigma_{i k}\left(T_{l}(v)\right) \\
& \times \operatorname{div}_{y} \zeta_{k}\left(T_{l}(v)\right) \partial_{x_{i}} \phi d x d t d y d s \\
&=\lim _{\varepsilon \downarrow 0} \int \sum_{k=1}^{K} \sum_{j=1}^{d} \operatorname{sign}_{\varepsilon}\left(T_{l}(u)-T_{l}(v)\right) \sigma_{j k}\left(T_{l}(u)\right) \\
& \quad \times \operatorname{div}_{x} \zeta_{k}\left(T_{l}(u)\right) \partial_{y_{j}} \phi d x d t d y d s \\
&-\lim _{\varepsilon \downarrow 0} \int \sum_{k=1}^{K} \sum_{i=1}^{d} \operatorname{sign}_{\varepsilon}\left(T_{l}(u)-T_{l}(v)\right) \sigma_{i k}\left(T_{l}(v)\right) \\
& \times \operatorname{div}_{y} \zeta_{k}\left(T_{l}(v)\right) \partial_{x_{i}} \phi d x d t d y d s .
\end{aligned}
$$

Invoking again the chain rule (D.3) in Definition 2.3 (keep in mind that $\operatorname{sign}_{\varepsilon}(\cdot)$ and the components of $\sigma(\cdot)$ belong to $C(\mathbf{R})$ ), we have for $1 \leq i, j \leq d$

$$
\begin{gathered}
\sum_{k=1}^{K} \operatorname{sign}_{\varepsilon}\left(T_{l}(u)-T_{l}(v)\right) \sigma_{j k}\left(T_{l}(u)\right) \operatorname{div}_{x} \zeta_{k}\left(T_{l}(u)\right) \\
=\sum_{i=1}^{d} \partial_{x_{i}} r_{i j}^{\varepsilon}\left(T_{l}(u), T_{l}(v)\right), \quad 1 \leq j \leq d, \\
\sum_{k=1}^{K} \operatorname{sign}_{\varepsilon}\left(T_{l}(u)-T_{l}(v)\right) \sigma_{i k}\left(T_{l}(v)\right) \operatorname{div}_{y} \zeta_{k}\left(T_{l}(v)\right) \\
=-\sum_{j=1}^{d} \partial_{y_{j}} r_{i j}^{\varepsilon}\left(T_{l}(u), T_{l}(v)\right), \quad 1 \leq i \leq d .
\end{gathered}
$$

Using (3.17) and integration by parts, we get

$$
\begin{aligned}
\lim _{\varepsilon \downarrow 0} E_{1} \geq- & \lim _{\varepsilon \downarrow 0} \int \sum_{i, j=1}^{d} r_{i j}^{\varepsilon}\left(T_{l}(u), T_{l}(v)\right) \partial_{x_{i} y_{j}}^{2} \phi d x d t d y d s \\
& -\lim _{\varepsilon \downarrow 0} \int \sum_{i, j=1}^{d} r_{i j}^{\varepsilon}\left(T_{l}(u), T_{l}(u)\right) \partial_{x_{i} y_{j}}^{2} \phi d x d t d y d s \\
= & -\int 2 \sum_{i, j=1}^{d} r_{i j}\left(T_{l}(u), T_{l}(u)\right) \partial_{x_{i} y_{j}}^{2} \phi d x d t d y d s .
\end{aligned}
$$

Consequently, adding this and (3.14) together yields (3.15). 
Summing up, sending $\varepsilon \downarrow 0$ in (3.13) gives

$$
\begin{gathered}
\int\left(I_{\text {time }}+I_{\text {conv }}+I_{\text {diff }}\right)(t, x, s, y) \omega_{\rho}\left(\frac{t-s}{2}, \frac{x-y}{2}\right) d x d t d y d s \\
-\int \operatorname{sign}\left(T_{l}(u)-T_{l}(v)\right)(F(t, x)-G(s, y)) \\
\times \omega_{\rho}\left(\frac{t-s}{2}, \frac{x-y}{2}\right) \varphi\left(\frac{t+s}{2}, \frac{x+y}{2}\right) d x d t d y d s \\
\geq-\mu_{l}^{u}\left((0, T) \times \mathbf{R}^{d}\right)-\mu_{l}^{v}\left((0, T) \times \mathbf{R}^{d}\right)
\end{gathered}
$$

where

$$
\begin{aligned}
& I_{\text {time }}(t, x, s, y)=\left|T_{l}(u(t, x))-T_{l}(v(s, y))\right|\left(\partial_{t}+\partial_{s}\right) \varphi\left(\frac{t+s}{2}, \frac{x+y}{2}\right), \\
& I_{\text {conv }}(t, x, s, y)=\sum_{i=1}^{d} q_{i}\left(T_{l}(u(t, x)), T_{l}(v(s, y))\right)\left(\partial_{x_{i}}+\partial_{y_{i}}\right) \varphi\left(\frac{t+s}{2}, \frac{x+y}{2}\right), \\
& I_{\text {diff }}(t, x, s, y)=\sum_{i, j=1}^{d} r_{i j}\left(T_{l}(u), T_{l}(v)\right)\left(\partial_{x_{i} x_{j}}^{2}+2 \partial_{x_{i} y_{j}}^{2}+\partial_{y_{i} y_{j}}^{2}\right) \varphi\left(\frac{t+s}{2}, \frac{x+y}{2}\right) .
\end{aligned}
$$

Let us introduce the change of variables

$$
\tilde{x}=\frac{x+y}{2}, \quad \tilde{t}=\frac{t+s}{2}, \quad z=\frac{x-y}{2}, \quad \tau=\frac{t-s}{2},
$$

which maps $(0, T) \times \mathbf{R}^{d} \times(0, T) \times \mathbf{R}^{d}$ into

$$
\Omega=\mathbf{R}^{d} \times \mathbf{R}^{d} \times\{(\tilde{t}, \tau) \mid 0 \leq \tilde{t}+\tau \leq T, \quad 0 \leq \tilde{t}-\tau \leq T\} .
$$

Observe that

$$
\left(\partial_{t}+\partial_{s}\right) \varphi\left(\frac{t+s}{2}, \frac{x+y}{2}\right)=\varphi_{\tilde{t}}(\tilde{t}, \tilde{x}), \quad\left(\nabla_{x}+\nabla_{y}\right) \varphi(t, x, s, y)=\nabla_{\tilde{x}} \varphi(\tilde{t}, \tilde{x}) .
$$

This change of variables diagonalizes also the operator $\nabla_{x x}+2 \nabla_{x y}+\nabla_{y y}$ :

$$
\left(\nabla_{x x}+2 \nabla_{x y}+\nabla_{y y}\right) \varphi\left(\frac{t+s}{2}, \frac{x+y}{2}\right)=\nabla_{\tilde{x} \tilde{x}} \varphi(\tilde{t}, \tilde{x}) .
$$

Keeping in mind that

$$
x=\tilde{x}+z, \quad y=\tilde{x}-z, \quad t=\tilde{t}+\tau, \quad s=\tilde{t}-\tau,
$$

we may now estimate (3.18) as

$$
\begin{aligned}
& \int_{\Omega}\left(I_{\text {time }}+I_{\text {conv }}-I_{\text {diff }}\right)(\tilde{t}, \tilde{x}, \tau, z) \omega_{\rho}(z) \delta_{\rho}(\tau) d \tilde{t} d \tilde{x} d \tau d z \\
& \geq-\int_{\Omega}|F(\tilde{t}+\tau, \tilde{x}+z)-G(\tilde{t}-\tau, \tilde{x}-z)| \omega_{\rho}(z) \delta_{\rho}(\tau) \varphi(\tilde{t}, \tilde{x}) d \tilde{x} d \tilde{t} d \tau d z \\
& \quad-\mu_{l}^{u}\left((0, T) \times \mathbf{R}^{d}\right)-\mu_{l}^{v}\left((0, T) \times \mathbf{R}^{d}\right),
\end{aligned}
$$


where

$$
\begin{aligned}
& I_{\text {time }}(\tilde{t}, \tilde{x}, \tau, z)=\left|T_{l}(u(\tilde{t}+\tau, \tilde{x}+z))-T_{l}(v(\tilde{t}-\tau, \tilde{x}-z))\right| \varphi_{\tilde{t}}(\tilde{t}, \tilde{x}), \\
& I_{\text {conv }}(\tilde{t}, \tilde{x}, \tau, z)=\sum_{i=1}^{d} q_{i}\left(T_{l}(u(\tilde{t}+\tau, \tilde{x}+z)), T_{l}(v(\tilde{t}-\tau, \tilde{x}-z))\right) \partial_{\tilde{x}_{i}} \varphi(\tilde{t}, \tilde{x}), \\
& I_{\text {diff }}(\tilde{t}, \tilde{x}, \tau, z)=\sum_{i, j=1}^{d} r_{i j}\left(T_{l}(u(\tilde{t}+\tau, \tilde{x}+z)), T_{l}(v(\tilde{t}-\tau, \tilde{x}-z))\right) \partial_{\tilde{x}_{i} \tilde{x}_{j}}^{2} \varphi .
\end{aligned}
$$

Sending $\rho \downarrow 0$ in (3.19) gives

$$
\begin{gathered}
\int\left(\left|T_{l}(u)-T_{l}(v)\right| \partial_{t} \varphi+\sum_{i=1}^{d} q_{i}\left(T_{l}(u), T_{l}(v)\right) \partial_{x_{i}} \varphi\right. \\
\left.+\sum_{i, j=1}^{d} r_{i j}\left(T_{l}(u), T_{l}(v)\right) \partial_{x_{i} x_{j}}^{2} \varphi\right) d x d t \\
\geq-\int|F(t, x)-G(t, x)| \varphi d x d t \\
\quad-\mu_{l}^{u}\left((0, T) \times \mathbf{R}^{d}\right)-\mu_{l}^{v}\left((0, T) \times \mathbf{R}^{d}\right) .
\end{gathered}
$$

By standard arguments (choosing a sequence of functions $0 \leq \varphi \leq 1$ from $\mathcal{D}\left((0, T) \times \mathbf{R}^{d}\right)$ that converges to $\mathbf{1}_{(0, t) \times \mathbf{R}^{d}}$ and using the initial conditions for $u, v$ in the sense of, say, (D.6) in Definition 2.3, it follows from (3.20) that for a.e. $t \in(0, T)$

$$
\begin{aligned}
& \int_{\mathbf{R}^{d}}\left|T_{l}(u(t, x))-T_{l}(v(t, x))\right| d x \\
& \leq \int_{\mathbf{R}^{d}}\left|T_{l}\left(u_{0}\right)-T_{l}\left(v_{0}\right)\right| d x+\int_{0}^{t} \int_{\mathbf{R}^{d}}|F(s, x)-G(s, x)| d x d s \\
& \quad+\mu_{l}^{u}\left((0, T) \times \mathbf{R}^{d}\right)+\mu_{l}^{v}\left((0, T) \times \mathbf{R}^{d}\right) .
\end{aligned}
$$

Equipped with (D.5) in Definition 2.3 for $u$ and $v$, sending $l \uparrow \infty$ in (3.21) yields finally the $L^{1}$ contraction property (3.4).

\section{EXISTENCE OF RENORMALIZED ENTROPY SOLUTION}

The purpose of this section is to prove the following theorem:

Theorem 4.1 (existence). Suppose (1.2), (1.3), and (1.4) hold. Then there exists at least one renormalized entropy solution $u$ of (1.1).

We divide the proof into two steps.

Step 1 (bounded data). Suppose the data $u_{0}$ and $F$ are bounded and integrable functions. Repeating the proof in [19] we find that there exists a unique entropy solution $u$ to (1.1) (interpreted in the sense of Definition 2.2 ), and this entropy solution can be constructed by the vanishing viscosity method [42]. For us it remains to prove that this entropy solution is also 
a renormalized entropy solution in the sense of Definition 2.3. To this end, let $u_{\rho}$ be the unique classical (say $C^{1,2}$ ) solution to the uniformly parabolic problem (see [42])

$$
\begin{aligned}
& \partial_{t} u_{\rho}+\operatorname{div} f\left(u_{\rho}\right)=\nabla \cdot\left(a\left(u_{\rho}\right) \nabla u_{\rho}\right)+\rho \Delta u_{\rho}+F, \quad \rho>0, \\
& u_{\rho}(x, 0)=u_{0}(x) .
\end{aligned}
$$

Equipped with the $\rho$-independent a priori estimates in [42], Chen and Perthame [19] prove

$$
u_{\rho} \rightarrow u \quad \text { a.e. and in } C\left(0, T ; L^{1}\left(\mathbf{R}^{d}\right)\right) \text { as } \rho \downarrow 0,
$$

where $u$ is the unique entropy solution to (1.1).

For any $C^{2}$ function $S$ and $\left(q_{i}^{S}\right)^{\prime}=S^{\prime} f_{i}^{\prime},\left(r_{i j}^{S}\right)^{\prime}=S^{\prime} a_{i j}$ for $1 \leq i, j \leq d$, multiplying the equation in (4.1) by $S^{\prime}\left(u_{\rho}\right)$ yields

$$
\begin{aligned}
\partial_{t} S\left(u_{\rho}\right) & +\sum_{i=1}^{d} \partial_{x_{i}} q_{i}^{S}\left(u_{\rho}\right)-\sum_{i, j=1}^{d} \partial_{x_{i} x_{j}}^{2} r_{i j}^{S}\left(u_{\rho}\right)-\rho \Delta S\left(u_{\rho}\right) \\
& -S^{\prime}\left(u_{\rho}\right) F\left(u_{\rho}\right)=-\left(n_{\rho}^{S^{\prime \prime}}+m_{\rho}^{S^{\prime \prime}}\right)(t, x),
\end{aligned}
$$

where the parabolic dissipation measure $n_{n, \rho}^{S^{\prime \prime}}(t, x)$ is defined by

$$
n_{\rho}^{S^{\prime \prime}}(t, x)=\sum_{k=1}^{K}\left(\sum_{i=1}^{d} \partial_{x_{i}} \zeta_{i k}^{S^{\prime \prime}}(u(t, x))\right)^{2} .
$$

and the entropy dissipation measure $m_{\rho}^{S^{\prime \prime}}(t, x)$ is defined by

$$
m_{\rho}^{S^{\prime \prime}}(t, x)=\rho S^{\prime \prime}\left(u_{\rho}\right)\left|\nabla u_{\rho}\right|^{2} .
$$

An easy approximation argument reveals that (4.3) continues to hold for any function $S \in W^{2, \infty}(\mathbf{R})$.

Inserting $S(u)=\frac{1}{l} \int_{0}^{u} T_{l}(\xi) \xi$ into (4.3) and then sending $l \downarrow 0$, we get the well known estimate

$$
\left\|u_{\rho}\right\|_{L^{\infty}\left(0, T ; L^{1}\left(\mathbf{R}^{d}\right)\right)} \leq\left\|u_{0}\right\|_{L^{1}\left(\mathbf{R}^{d}\right)}+\|F\|_{L^{1}\left((0, T) \times \mathbf{R}^{d}\right)} .
$$

We need to derive some additional a priori estimates (involving (2.4)) that are independent of $\rho$ and $\left\|u_{0}\right\|_{L^{\infty}\left(\mathbf{R}^{d}\right)},\|F\|_{L^{\infty}\left((0, T) \times \mathbf{R}^{d}\right)}$.

Lemma 4.1. For any $l>0$, we have

$$
\int_{(0, T) \times \mathbf{R}^{d}}\left(\sum_{k=1}^{K}\left(\sum_{i=1}^{d} \partial_{x_{i}} \zeta_{i k}\left(T_{l}\left(u_{\rho}\right)\right)\right)^{2}+\rho\left|\nabla T_{l}\left(u_{\rho}\right)\right|^{2}\right) d x d t \leq C_{l},
$$

for some constant $C_{l}$ that is independent of $\rho$ but not $l$. More precisely,

$$
C_{l}=l\left(\left\|u_{0}\right\|_{L^{1}\left(\mathbf{R}^{d}\right)}+\|F\|_{L^{1}\left((0, T) \times \mathbf{R}^{d}\right)}\right) .
$$


Proof. Introduce the function

$$
S(u)=\int_{0}^{u} T_{l}(\xi) d \xi= \begin{cases}\frac{|u|^{2}}{2}, & \text { if }|u| \leq l \\ l|u|-\frac{l^{2}}{2}, & \text { if }|u|>l .\end{cases}
$$

Choosing this $S(\cdot)$ in $(4.3)$, we derive

$$
\begin{array}{rl}
\int_{\mathbf{R}^{d}} & S\left(u_{\rho}(T, x)\right) d x-\int_{\mathbf{R}^{d}} S\left(u_{0}(x)\right) d x-\int_{0}^{T} \int_{\mathbf{R}^{d}} F\left(u_{\rho}\right) T_{l}\left(u_{\rho}\right) d x d t \\
= & -\int_{(0, T) \times \mathbf{R}^{d}}\left(\sum_{k=1}^{K}\left(\sum_{i=1}^{d} \partial_{x_{i}} \zeta_{i k}\left(T_{l}\left(u_{\rho}\right)\right)\right)^{2}+\rho\left|\nabla T_{l}\left(u_{\rho}\right)\right|^{2}\right) d x d t .
\end{array}
$$

From the nonnegativity of $S(\cdot)$, it follows from (4.5)

$$
\begin{gathered}
\int_{(0, T) \times \mathbf{R}^{d}}\left(\sum_{k=1}^{K}\left(\sum_{i=1}^{d} \partial_{x_{i}} \zeta_{i k}\left(T_{l}\left(u_{\rho}\right)\right)\right)^{2}+\rho\left|\nabla T_{l}\left(u_{\rho}\right)\right|^{2}\right) d x d t \\
\leq \int_{\mathbf{R}^{d}} S\left(u_{0}(x)\right) d x+\int_{0}^{T} \int_{\mathbf{R}^{d}} F\left(u_{\rho}\right) T_{l}\left(u_{\rho}\right) d x d t .
\end{gathered}
$$

Since for all $u \in \mathbf{R},\left|T_{l}(u)\right| \leq l$ and $0 \leq S(u) \leq l|u|$, we deduce from (4.6)

$$
\begin{aligned}
& \int_{(0, T) \times \mathbf{R}^{d}}\left(\sum_{k=1}^{K}\left(\sum_{i=1}^{d} \partial_{x_{i}} \zeta_{i k}\left(T_{l}\left(u_{\rho}\right)\right)\right)^{2}+\rho\left|\nabla T_{l}\left(u_{\rho}\right)\right|^{2}\right) d x d t \\
& \leq l\left(\left\|u_{0}\right\|_{L^{1}\left(\mathbf{R}^{d}\right)}+\|F\|_{L^{1}\left((0, T) \times \mathbf{R}^{d}\right)}\right),
\end{aligned}
$$

which completes the proof of Lemma 4.1.

Lemma 4.2. For any $l>0$ and any $\delta>0$,

$$
\frac{1}{\delta} \int_{\left\{l<\left|u_{\rho}\right|<l+\delta\right\}}\left(\sum_{k=1}^{K}\left(\sum_{i=1}^{d} \partial_{x_{i}} \zeta_{i k}\left(u_{\rho}\right)\right)^{2}+\rho\left|\nabla u_{\rho}\right|^{2}\right) d x d t \leq \mathcal{E}(l),
$$

for some bounded function $\mathcal{E}(\cdot)$ on $\mathbf{R}_{+}$that is independent of $n, \rho, \delta$ and satisfies

$$
\lim _{l \uparrow \infty} \mathcal{E}(l)=0,
$$

If the data $u_{0}, F$ are bounded and

$$
l>M:=\left\|u_{0}\right\|_{L^{\infty}\left(\mathbf{R}^{d}\right)}+\|F\|_{L^{\infty}\left((0, T) \times \mathbf{R}^{d}\right)},
$$

then $\mathcal{E}(l)=0$. 
Proof. Let us define the function $S(\cdot)$ by $S(0)=0$ and

$$
S^{\prime}(u)=\frac{1}{\delta}\left(T_{l+\delta}(u)-T_{l}(u)\right)= \begin{cases}-1, & u<-l-\delta, \\ \frac{-u-l}{\delta}, & -l-\delta<u<-l, \\ 0, & -l<u<l, \\ \frac{u-l}{\delta}, & l<u<l+\delta, \\ 1, & u>l+\delta .\end{cases}
$$

Inserting this $S$ into (4.3) gives

$$
\begin{aligned}
& \frac{1}{\delta} \int_{\left\{l<\left|u_{\rho}\right|<l+\delta\right\}}\left(\sum_{k=1}^{K}\left(\sum_{i=1}^{d} \partial_{x_{i}} \zeta_{i k}\left(u_{\rho}\right)\right)^{2}+\rho\left|\nabla u_{\rho}\right|^{2}\right) d x d t \\
& \quad \leq \int_{\left\{\left|u_{0}\right|>l\right\}}\left|u_{0}\right| d x+\int_{\left\{\left|u_{\rho}\right|>l\right\}}|F| d x d t:=\mathcal{E}(l) .
\end{aligned}
$$

Since $u_{0} \in L^{1}\left(\mathbf{R}^{d}\right), F \in L^{1}\left((0, T) \times \mathbf{R}^{d}\right)$, and, thanks to (4.4), $u_{\rho}$ is uniformly (in $\rho$ ) bounded in $L^{1}\left((0, T) \times \mathbf{R}^{d}\right)$, we have $\mathcal{E}(l) \rightarrow 0$ as $l \uparrow \infty$.

If the data $u_{0}, F$ are bounded functions, then it is well known that $\left\|u_{\rho}\right\|_{L^{\infty}\left((0, T) \times \mathbf{R}^{d}\right)} \leq M$, where $M$ is defined in (4.8). We observe that if $l>M$, then $S\left(u_{0}\right)=0$ and $S^{\prime}\left(u_{\rho}\right)=0$. Hence we deduce $\mathcal{E}(l)=0$.

Let us choose a particular $S=S_{\eta, h}$ in (4.3) of the form

$$
\begin{array}{ll}
S_{\eta, h}(0)=0, & S_{\eta, h}^{\prime}=\eta^{\prime} h^{\prime}, \\
\eta \in C^{2}(\mathbf{R}), & \eta^{\prime \prime} \geq 0, \quad\left|\eta^{\prime}\right| \leq K, \\
h \in C^{2}(\mathbf{R}), & \operatorname{supp}\left(h^{\prime}\right) \subset[-l, l] .
\end{array}
$$

This gives

$$
\begin{gathered}
\partial_{t} S_{\eta, h}\left(u_{\rho}\right)+\sum_{i=1}^{d} \partial_{x_{i}} q_{i}^{S_{\eta, h}}\left(u_{\rho}\right)-\sum_{i, j=1}^{d} \partial_{x_{i} x_{j}}^{2} r_{i j}^{S_{\eta, h}}\left(u_{\rho}\right)-\rho \Delta S_{\eta, h}\left(u_{\rho}\right) \\
-S_{\eta, h}^{\prime}\left(u_{\rho}\right) F\left(u_{\rho}\right)=-\left(n_{\rho}^{\eta^{\prime \prime} h^{\prime}}+\mu_{\rho}^{\eta^{\prime} h^{\prime \prime}}\right)(t, x)
\end{gathered}
$$

where

$$
\begin{aligned}
\mu_{\rho}^{\eta^{\prime} h^{\prime \prime}}(t, x) & :=-\left(n_{\rho}^{\eta^{\prime} h^{\prime \prime}}+m_{\rho}^{\eta^{\prime} h^{\prime \prime}}\right)(t, x) \\
& =-\eta^{\prime}\left(u_{\rho}\right) h^{\prime \prime}\left(u_{\rho}\right)\left(\sum_{k=1}^{K}\left(\sum_{i=1}^{d} \partial_{x_{i}} \zeta_{i k}\left(u_{\rho}\right)\right)^{2}+\rho\left|\nabla u_{\rho}\right|^{2}\right)
\end{aligned}
$$

Let $h_{l, \delta}: \mathbf{R} \rightarrow \mathbf{R}$ denote the function defined by $h_{l, \delta}(0)=0$ and

$$
h_{l, \delta}^{\prime}(u)= \begin{cases}1, & |u|<l, \\ \frac{l+\delta-|u|}{\delta}, & l<|u|<l+\delta, \\ 0, & |u|>l+\delta\end{cases}
$$


Clearly,

$$
h_{l, \delta}(u) \rightarrow T_{l}(u), \quad h_{l, \delta}^{\prime}(u) \rightarrow \mathbf{1}_{\{|u|<l\}},
$$

for any $u \in \mathbf{R}$. The idea is to choose $h=h_{n, l}$ in (4.10) and then let $\delta \downarrow 0$. To this end, let us first define the Radon measure $\mu_{l, n, \rho, \delta}^{K}$ on $(0, T) \times \mathbf{R}^{d}$ by

$$
d \mu_{l, \rho, \delta}^{K}(t, x):=\frac{K}{\delta} \mathbf{1}_{\left\{l<\left|u_{\rho}\right|<l+\delta\right\}}\left(\sum_{k=1}^{K}\left(\sum_{i=1}^{d} \partial_{x_{i}} \zeta_{i k}\left(u_{\rho}\right)\right)^{2}+\rho\left|\nabla u_{\rho}\right|^{2}\right) d x d t,
$$

that is, for any Borel set $E \subset(0, T) \times \mathbf{R}^{d}$,

$$
\mu_{l, \rho, \delta}^{K}(\mathcal{E})=\frac{K}{\delta} \int_{E \cap\left\{l<\left|u_{\rho}\right|<l+\delta\right\}}\left(\sum_{k=1}^{K}\left(\sum_{i=1}^{d} \partial_{x_{i}} \zeta_{i k}\left(u_{\rho}\right)\right)^{2}+\rho\left|\nabla u_{\rho}\right|^{2}\right) d x d t .
$$

Then, by Lemma 4.2 ,

$$
\mu_{l, \rho, \delta}^{K}\left((0, T) \times \mathbf{R}^{d}\right) \leq \mathcal{E}(l) .
$$

Consequently, we may assume that

$$
\begin{aligned}
& \mu_{l, \rho, \delta}^{K} \stackrel{\star}{\rightarrow} \mu_{l, \rho}^{K} \quad \text { in the sense of measures on }(0, T) \times \mathbf{R}^{d} \text { as } \delta \downarrow 0, \\
& \mu_{l, \rho}^{K} \stackrel{\star}{\rightarrow} \mu_{l}^{K} \quad \text { in the sense of measures on }(0, T) \times \mathbf{R}^{d} \rho \downarrow 0,
\end{aligned}
$$

for some nonnegative bounded Radon measure $\mu_{l}^{K}$ satisfying

$$
\mu_{l}^{K}\left((0, T) \times \mathbf{R}^{d}\right) \leq \mathcal{E}(l) \rightarrow 0 \quad \text { as } l \uparrow \infty .
$$

For any $0 \leq \phi \in \mathcal{D}\left((0, T) \times \mathbf{R}^{d}\right)$, thanks to (4.11) and the convexity of $\eta$,

$$
\begin{aligned}
& \lim _{\delta \downarrow 0} \int_{(0, T) \times \mathbf{R}^{d}} n_{\rho}^{\eta^{\prime \prime} h_{l, \delta}^{\prime}}(t, x) \phi d x d t \\
& \quad=\int_{(0, T) \times \mathbf{R}^{d}} \eta^{\prime \prime}\left(u_{\rho}\right) \mathbf{1}_{\left\{\left|u_{\rho}\right|<l\right\}}\left(\sum_{k=1}^{K}\left(\sum_{i=1}^{d} \partial_{x_{i}} \zeta_{i k}\left(u_{\rho}\right)\right)^{2}+\rho\left|\nabla u_{\rho}\right|^{2}\right) \phi d x d t \\
& \quad \geq \int_{(0, T) \times \mathbf{R}^{d}} \eta^{\prime \prime}\left(T_{l}\left(u_{\rho}\right)\right) \sum_{k=1}^{K}\left(\sum_{i=1}^{d} \partial_{x_{i}} \zeta_{i k}\left(T_{l}\left(u_{\rho}\right)\right)\right)^{2} \phi d x d t .
\end{aligned}
$$

Again because of (4.11), it can be easily checked that as $\delta \downarrow 0$ (recall $q^{\prime}=\eta^{\prime} f^{\prime}$ and $\left.r^{\prime}=\eta^{\prime} a\right)$

$$
\begin{aligned}
S_{\eta, h_{l, \delta}}(u) & \rightarrow \eta\left(T_{l}(u)\right), & S_{\eta, h_{l, \delta}}^{\prime}(u) & \rightarrow \eta^{\prime}\left(T_{l}(u)\right), \\
q^{S_{\eta, h_{l}, \delta}}(u) & \rightarrow q\left(T_{l}(u)\right), & r^{S_{\eta, h_{l}, \delta}}(u) & \rightarrow r\left(T_{l}(u)\right),
\end{aligned}
$$

for any $u \in \mathbf{R}$. 
Inserting $h=h_{l, \delta}$ into (4.10) and using $\left|\eta^{\prime}\right| \leq K$, (4.12), (4.14), (4.15) when sending $\delta \downarrow 0$, we get

$$
\begin{aligned}
& \partial_{t} \eta\left(T_{l}\left(u_{\rho}\right)\right)+ \sum_{i=1}^{d} \partial_{x_{i}} q_{i}\left(T_{l}\left(u_{\rho}\right)\right)-\sum_{i, j=1}^{d} \partial_{x_{i} x_{j}}^{2} r_{i j}\left(T_{l}\left(u_{\rho}\right)\right) \\
&-\rho \Delta \eta\left(T_{l}\left(u_{\rho}\right)\right)-\eta^{\prime}\left(T_{l}\left(u_{\rho}\right)\right) F \\
& \leq-\eta^{\prime \prime}\left(T_{l}\left(u_{\rho}\right)\right) \sum_{k=1}^{K}\left(\sum_{i=1}^{d} \partial_{x_{i}} \zeta_{i k}\left(u_{\rho}\right)\right)^{2}+\mu_{l, \rho}^{K} \quad \text { in } \mathcal{D}^{\prime}\left((0, T) \times \mathbf{R}^{d}\right) .
\end{aligned}
$$

We now want to send $\rho \downarrow 0$ in (4.16). To pass to the limit in the first term on the right-hand side of (4.16) we employ a standard lower semicontinuity result found, e.g., in [1]. For any $0 \leq \phi \in \mathcal{D}\left((0, T) \times \mathbf{R}^{d}\right)$ we have

$$
\begin{gathered}
\liminf _{\rho \downarrow 0} \int_{(0, T) \times \mathbf{R}^{d}} \eta^{\prime \prime}\left(T_{l}\left(u_{\rho}\right)\right) \sum_{k=1}^{K}\left(\sum_{i=1}^{d} \partial_{x_{i}} \zeta_{i k}\left(T_{l}\left(u_{\rho}\right)\right)\right)^{2} \phi d x d t \\
\geq \int_{(0, T) \times \mathbf{R}^{d}} \eta^{\prime \prime}\left(T_{l}(u)\right) \sum_{k=1}^{K}\left(\sum_{i=1}^{d} \partial_{x_{i}} \zeta_{i k}\left(T_{l}(u)\right)\right)^{2} \phi d x d t .
\end{gathered}
$$

Equipped with (4.17) and (4.12), passing to the limit $\rho \downarrow 0$ in (4.16) yields

$$
\begin{gathered}
\int_{(0, T) \times \mathbf{R}^{d}}\left(\eta\left(T_{l}(u)\right) \partial_{t} \phi+\sum_{i=1}^{d} q_{i}\left(T_{l}(u)\right) \partial_{x_{i}} \phi+\sum_{i, j=1}^{d} r_{i j}\left(T_{l}(u)\right) \partial_{x_{i} x_{j}}^{2} \phi\right) d x d t \\
+\int_{(0, T) \times \mathbf{R}^{d}} \eta^{\prime}\left(T_{l}(u)\right) F \phi d x d t \\
\geq \int_{(0, T) \times \mathbf{R}^{d}} \eta^{\prime \prime}\left(T_{l}(u)\right) \sum_{k=1}^{K}\left(\sum_{i=1}^{d} \partial_{x_{i}} \zeta_{i k}\left(T_{l}(u)\right)\right)^{2} \phi d x d t \\
\quad-\int_{(0, T) \times \mathbf{R}^{d}} \phi d \mu_{l}^{K}(t, x), \quad \forall \phi \in \mathcal{D}\left((0, T) \times \mathbf{R}^{d}\right), \phi \geq 0 .
\end{gathered}
$$

It remains to prove that the chain rule (D.3) in Definition 2.3 holds. For any $\psi \in C(\mathbf{R})$, the classical chain rule gives for $k=1, \ldots, K$

$$
\sum_{i=1}^{d} \partial_{x_{i}} \zeta_{i k}^{\psi}\left(T_{l}\left(u_{\rho}\right)\right)=\psi\left(T_{l}\left(u_{\rho}\right)\right) \sum_{i=1}^{d} \partial_{x_{i}} \zeta_{i k}\left(T_{l}\left(u_{\rho}\right)\right), \quad \forall l>0 .
$$

As in [19], the proof is to observe that this equality continues to hold in the limit as $\rho \downarrow 0$ since $u_{\rho}$ converges strongly and $\sum_{i=1}^{d} \partial_{x_{i}} \zeta_{i k}\left(T_{l}\left(u_{\rho}\right)\right)$ weakly.

Step 2 (unbounded data). Suppose the data $u_{0}$ and $F$ satisfy (1.2). For $n>1$, introduce the truncated data $u_{0, n}=T_{n}\left(u_{0}\right)$ and $F_{n}=T_{n}(F)$. We have $u_{0, n} \rightarrow u_{0}, F_{n} \rightarrow F$ in $L^{1}$ as $n \uparrow \infty$. Thanks to the $L^{1}$ contraction 
property of the solution operator to (4.1), the following estimate holds for a.e. $t \in(0, T)$ :

$$
\begin{aligned}
& \left\|u_{n^{\prime}}(\cdot, t)-u_{n}(\cdot, t)\right\|_{L^{1}\left(\mathbf{R}^{d}\right)} \\
& \quad \leq\left\|u_{0, n^{\prime}}-u_{0, n}\right\|_{L^{1}\left(\mathbf{R}^{d}\right)}+\int_{0}^{t}\left\|F_{n^{\prime}}(s, \cdot)-F_{n}(s, \cdot)\right\|_{L^{1}\left(\mathbf{R}^{d}\right)} d s \rightarrow 0,
\end{aligned}
$$

as $n, n^{\prime} \rightarrow \infty$. Hence $\left\{u_{n}\right\}_{n>1}$ is a Cauchy sequence in $C\left(0, T ; L^{1}\left(\mathbf{R}^{d}\right)\right)$ and has a limit point $u$. From Step 1 we know that each $u_{n}$ is a renormalized entropy solution of (1.1) with $u_{0}$ and $F$ replaced by $u_{0, n}$ and $F_{n}$, respectively. Denote by $\mu_{l, n}^{K}$ the corresponding renormalization measure. Lemma 4.1 and (4.13) imply that the following $n$-independent a priori estimates hold for each $l>0$ :

$$
\begin{aligned}
& \left\|u_{n}\right\|_{L^{\infty}\left(0, T ; L^{1}\left(\mathbf{R}^{d}\right)\right)} \leq\left\|u_{0}\right\|_{L^{1}\left(\mathbf{R}^{d}\right)}+\|F\|_{L^{1}\left((0, T) \times \mathbf{R}^{d}\right)}, \\
& \sum_{k=1}^{K}\left(\operatorname{div} \zeta_{k}\left(T_{l}(u)\right)\right)^{2}=\sum_{k=1}^{K}\left(\sum_{i=1}^{d} \partial_{x_{i}} \zeta_{i k}\left(T_{l}\left(u_{n}\right)\right)\right)^{2} \leq C_{l}, \\
& \mu_{l, n}^{K}\left((0, T) \times \mathbf{R}^{d}\right) \leq \int_{\left\{\left|u_{0}\right|>l\right\}}\left|u_{0}\right| d x+\int_{\left\{\left|u_{n}\right|>l\right\}}|F| d x d t,
\end{aligned}
$$

for some constant $C_{l}$ that depends on $l$ but not $n$. Equipped with these estimates and the strong convergence $u_{n} \rightarrow u$, we can repeat the steps in the above limiting process for the viscous approximations $\left\{u_{\rho}\right\}_{\rho>0}$ and prove that the limit point $u$ of $\left\{u_{n}\right\}_{n>1}$ is a renormalized entropy solution of (1.1) with a renormalization measure $\mu_{l}^{u, K}$ being a limit point of $\left\{\mu_{l, n}^{K}\right\}_{n>1}$. This completes the proof of Theorem 4.1.

\section{REFERENCES}

[1] L. Ambrosio, N. Fusco, and D. Pallara. Functions of bounded variation and free discontinuity problems. Oxford Mathematical Monographs. The Clarendon Press Oxford University Press, New York, 2000.

[2] D. Aregba-Driollet, R. Natalini, and S. Tang. Explicit diffusive kinetic schemes for nonlinear degenerate parabolic systems. Preprint, 2000.

[3] Y. Atik and J. M. Rakotoson. Local T-sets and renormalized solutions of degenerate quasilinear elliptic equations with an $L^{1}$-datum. Adv. Differential Equations, 1(6):965-988, 1996.

[4] P. Bénilan, L. Boccardo, T. Gallouët, R. Gariepy, M. Pierre, and J. L. Vázquez. An $L^{1}$-theory of existence and uniqueness of solutions of nonlinear elliptic equations. Ann. Scuola Norm. Sup. Pisa Cl. Sci. (4), 22(2):241-273, 1995.

[5] P. Bénilan, J. Carrillo, and P. Wittbold. Renormalized entropy solutions of scalar conservation laws. Ann. Scuola Norm. Sup. Pisa Cl. Sci. (4), 29(2):313-327, 2000.

[6] P. Bénilan and H. Touré. Sur l'équation générale $u_{t}=a\left(\cdot, u, \phi(\cdot, u)_{x}\right)_{x}+v$ dans $L^{1}$. II. Le problème d'évolution. Ann. Inst. H. Poincaré Anal. Non Linéaire, 12(6):727-761, 1995.

[7] D. Blanchard and F. Murat. Renormalised solutions of nonlinear parabolic problems with $L^{1}$ data: existence and uniqueness. Proc. Roy. Soc. Edinburgh Sect. A, 127(6):1137-1152, 1997. 
[8] D. Blanchard, F. Murat, and H. Redwane. Existence and uniqueness of a renormalized solution for a fairly general class of nonlinear parabolic problems. J. Differential Equations, 177(2):331-374, 2001.

[9] D. Blanchard and H. Redwane. Renormalized solutions for a class of nonlinear evolution problems. J. Math. Pures Appl. (9), 77(2):117-151, 1998.

[10] L. Boccardo, J. I. Diaz, D. Giachetti, and F. Murat. Existence of a solution for a weaker form of a nonlinear elliptic equation. In Recent advances in nonlinear elliptic and parabolic problems (Nancy, 1988), volume 208 of Pitman Res. Notes Math. Ser., pages 229-246. Longman Sci. Tech., Harlow, 1989.

[11] L. Boccardo, D. Giachetti, J. I. Diaz, and F. Murat. Existence and regularity of renormalized solutions for some elliptic problems involving derivatives of nonlinear terms. J. Differential Equations, 106(2):215-237, 1993.

[12] F. Bouchut, F. R. Guarguaglini, and R. Natalini. Diffusive BGK approximations for nonlinear multidimensional parabolic equations. Indiana Univ. Math. J., 49(2):723749, 2000.

[13] R. Bürger, S. Evje, and K. H. Karlsen. On strongly degenerate convection-diffusion problems modeling sedimentation-consolidation processes. J. Math. Anal. Appl., 247(2):517-556, 2000 .

[14] J. Carrillo. On the uniqueness of the solution of the evolution dam problem. Nonlinear Anal., 22(5):573-607, 1994.

[15] J. Carrillo. Entropy solutions for nonlinear degenerate problems. Arch. Rational Mech. Anal., 147(4):269-361, 1999.

[16] J. Carrillo and P. Wittbold. Uniqueness of renormalized solutions of degenerate elliptic-parabolic problems. J. Differential Equations, 156(1):93-121, 1999.

[17] G.-Q. Chen and E. DiBenedetto. Stability of entropy solutions to the Cauchy problem for a class of nonlinear hyperbolic-parabolic equations. SIAM J. Math. Anal., 33(4):751-762 (electronic), 2001.

[18] G.-Q. Chen and K. H. Karlsen. $L^{1}$ framework for continuous dependence and error estimates for quasi-linear degenerate parabolic equations. Preprint, 2002.

[19] G.-Q. Chen and B. Perthame. Well-posedness for non-isotropic degenerate parabolichyperbolic equations. Annales de l'Institut Henri Poincaré: Analyse Non Linéaire. To appear.

[20] B. Cockburn and G. Gripenberg. Continuous dependence on the nonlinearities of solutions of degenerate parabolic equations. J. Differential Equations, 151(2):231251, 1999.

[21] G. Dal Maso, F. Murat, L. Orsina, and A. Prignet. Renormalized solutions of elliptic equations with general measure data. Ann. Scuola Norm. Sup. Pisa Cl. Sci. (4), 28(4):741-808, 1999.

[22] R. J. DiPerna and P.-L. Lions. On the Fokker-Planck-Boltzmann equation. Comm. Math. Phys., 120(1):1-23, 1988.

[23] R. J. DiPerna and P.-L. Lions. On the Cauchy problem for Boltzmann equations: global existence and weak stability. Ann. of Math. (2), 130(2):321-366, 1989.

[24] R. J. DiPerna and P.-L. Lions. Ordinary differential equations, transport theory and Sobolev spaces. Invent. Math., 98(3):511-547, 1989.

[25] M. S. Espedal and K. H. Karlsen. Numerical solution of reservoir flow models based on large time step operator splitting algorithms. In Filtration in Porous Media and Industrial Applications (Cetraro, Italy, 1998), volume 1734 of Lecture Notes in Mathematics, pages 9-77. Springer, Berlin, 2000.

[26] S. Evje and K. H. Karlsen. Monotone difference approximations of $B V$ solutions to degenerate convection-diffusion equations. SIAM J. Numer. Anal., 37(6):1838-1860 (electronic), 2000. 
[27] R. Eymard, T. Gallouët, R. Herbin, and A. Michel. Convergence of a finite volume scheme for nonlinear degenerate parabolic equations. Numer. Math., 92(1):41-82, 2002.

[28] D. Gilbarg and N. S. Trudinger. Elliptic partial differential equations of second order, volume 224 of Grundlehren der Mathematischen Wissenschaften [Fundamental Principles of Mathematical Sciences]. Springer-Verlag, Berlin, second edition, 1983.

[29] K. H. Karlsen and M. Ohlberger. A note on the uniqueness of entropy solutions of nonlinear degenerate parabolic equations. J. Math. Anal. Appl., 275(1):439-458, 2002 .

[30] K. H. Karlsen and N. H. Risebro. Convergence of finite difference schemes for viscous and inviscid conservation laws with rough coefficients. M2AN Math. Model. Numer. Anal., 35(2):239-269, 2001.

[31] K. H. Karlsen and N. H. Risebro. On the uniqueness and stability of entropy solutions of nonlinear degenerate parabolic equations with rough coefficients. Discrete Contin. Dyn. Syst., 9(5):1081-1104, 2003.

[32] S. N. Kružkov. First order quasi-linear equations in several independent variables. Math. USSR Sbornik, 10(2):217-243, 1970.

[33] P.-L. Lions. Mathematical topics in fluid mechanics. Vol. 1, volume 3 of Oxford Lecture Series in Mathematics and its Applications. Oxford University Press, New York, 1996.

[34] C. Mascia, A. Porretta, and A. Terracina. Nonhomogeneous dirichlet problems for degenerate parabolic-hyperbolic equations. Arch. Ration. Mech. Anal., 163:87-124, 2002.

[35] A. Michel and J. Vovelle. Entropy formulation for parabolic degenerate equations with general Dirichlet boundary conditions and application to the convergence of FV methods. Preprint, 2002.

[36] M. Ohlberger. A posteriori error estimates for vertex centered finite volume approximations of convection-diffusion-reaction equations. M2AN Math. Model. Numer. Anal., 35(2):355-387, 2001.

[37] B. Perthame. Kinetic formulation of conservation laws, volume 21 of Oxford Lecture Series in Mathematics and its Applications. Oxford University Press, New York, 2002.

[38] J.-M. Rakotoson. Uniqueness of renormalized solutions in a $T$-set for the $L^{1}$ data problem and the link between various formulations. Indiana Univ. Math. J., 43(2):685-702, 1994.

[39] É. Rouvre and G. Gagneux. Solution forte entropique de lois scalaires hyperboliquesparaboliques dégénérées. C. R. Acad. Sci. Paris Sér. I Math., 329(7):599-602, 1999.

[40] T. Tassa. Uniqueness of piecewise smooth weak solutions of multidimensional degenerate parabolic equations. J. Math. Anal. Applic., 210:598-608, 1997.

[41] A. I. Vol'pert. The spaces BV and quasi-linear equations. Math. USSR Sbornik, $2(2): 225-267,1967$.

[42] A. I. Vol'pert and S. I. Hudjaev. Cauchy's problem for degenerate second order quasilinear parabolic equations. Math. USSR Sbornik, 7(3):365-387, 1969.

[43] Z. Wu and J. Yin. Some properties of functions in $B V_{x}$ and their applications to the uniqueness of solutions for degenerate quasilinear parabolic equations. Northeastern Math. J., 5(4):395-422, 1989. 
(Mostafa Bendahmane)

Department of Mathematics

UNIVERSITY OF BERGEN

Johs. Brunsgt. 12

N-5008 Bergen, NorWAY

E-mail address: mostafab@math.uio.no

(Kenneth Hvistendahl Karlsen)

Department of Mathematics

UNIVERSITY OF BERGEN

Johs. Brunsgt. 12

N-5008 BERGEN, NoRWAY

AND

Centre of Mathematics for Applications

Department of Mathematics

UNIVERSITY OF OSLO

P.O. BOX 1053, BLINDERN

N-0316 Oslo, Norway

E-mail address: kennethk@mi.uib.no

$U R L$ : http://www.mi.uib.no/ ${ }^{\sim}$ kennethk/ 